\title{
BMJ Open Risk of behaviour suggestive of opioid abuse: a protocol for a systematic review of validated assessment tools
}

\author{
Sheila Raposo Galindo, ${ }^{1}$ Tatiana de Paula Santana da Silva, ${ }^{2}$ \\ Manoel Henrique da Nóbrega Marinho, ${ }^{3}$ Carlos Eduardo de Souza Leão Ribeiro, ${ }^{4}$ \\ Murilo Duarte da Costa Lima, ${ }^{5}$ Selene Cordeiro Vasconcelos ${ }^{6}$
}

To cite: Galindo SR, Silva TdPSda, Marinho MHdN, et al. Risk of behaviour suggestive of opioid abuse: a protocol for a systematic review of validated assessment tools. BMJ Open 2018;8:e021948. doi:10.1136/ bmjopen-2018-021948

- Prepublication history for this paper is available online. To view these files, please visit the journal online (http://dx.doi. org/10.1136/bmjopen-2018021948).

Received 30 January 2018

Revised 3 July 2018

Accepted 23 August 2018
Check for updates

(c) Author(s) (or their employer(s)) 2018. Re-use permitted under CC BY-NC. No commercial re-use. See rights and permissions. Published by BMJ.

For numbered affiliations see end of article.

Correspondence to Dr Sheila Raposo Galindo; sheilagalindo@hotmail.com

\section{ABSTRACT}

Introduction Opioid use patterns of individuals with noncancer pain are influenced by the behavioural dynamics of the individual in managing and properly following the prescription. The use of assessment tools for measuring the risk of behaviour suggestive of opioid abuse is important for health professionals who provide care to individuals with non-cancer pain. The aim of the proposed review is to analyse the psychometric properties of tools for measuring the risk of behaviour suggestive of opioid abuse in adults with non-cancer pain.

Methods and analysis The review process will be based on the Preferred Reporting Items for Systematic Review and Meta-Analysis Protocols. The ConsensusBased Standards for the Selection of Health Measurement Instruments will be used to analyse the assessment tools. Two independent reviewers will perform the literature search and analysis procedures. Searches will be performed on PubMed, Web of Science, Cochrane, Scopus, and Cumulative Index to Nursing and Allied Health Literature databases, and the 'snowball' strategy will be employed. The inclusion criteria will be (1) validation studies, (2) assessment tools designed exclusively for measuring the risk of behaviour suggestive of opioid abuse and (3) assessment tools designed for evaluation of adults with chronic non-cancer pain. The titles and abstracts of the studies retrieved from the databases will be analysed for the preselection of articles, which will be submitted to a full-text analysis to define the final sample. Divergence of opinion between two reviewers will be resolved by consulting a third reviewer.

Ethics and dissemination The review will offer an overview of assessment tools available for measuring the risk of behaviour suggestive of opioid abuse, which is relevant to reducing the risk of deaths due to abusive consumption and for clinical management of adults with chronic non-cancer pain.

PROSPERO registration number CRD42018081577.

\section{INTRODUCTION}

The consumption of opioids and the risk of improper use in populations with chronic non-cancer pain have generated considerable discussion in recent years. The improper use of opioids can result in serious consequences
Strengths and limitations of this study

- The review broadens the understanding on the risk of behaviour suggestive of opioid abuse among adults with chronic non-cancer pain.

- It provides an overview of assessment tools for evaluating the opioid consumption pattern of adults with chronic non-cancer pain.

- It provides evidence of the best assessment tools for measuring this health phenomenon to assist in the decision-making processes of health professionals who provide care to such patients.

- It assists in the development of therapeutic guidelines for the management of opioid consumption in adults with chronic non-cancer pain.

- Limitations may be related to the subjectivity of the researchers with regard to delineating evidence focused on specific gaps in knowledge in the field of interest.

to one's health, contributing to the development of dependency on these drugs. ${ }^{1}$ It is estimated that up to $60 \%$ of patients with chronic pain who take opioids are susceptible to abusive use, commonly in the form of excessive consumption. ${ }^{2}$ Another study reports that $61.8 \%$ of patients had chronic pain prior to their first diagnosis of an opioid use disorder. ${ }^{3}$

The risk of behaviour suggestive of opioid abuse constitutes a predictor of the development of a substance use disorder, which is a real possibility for individuals with chronic non-cancer pain and a considerable concern for health professionals. ${ }^{4}$ Opioid use patterns of individuals with non-cancer pain are influenced by the behavioural dynamics of the individual in managing and properly following the prescription, as well as the skills of health professionals regarding the identification of risk and protection factors of opioid abuse by these individuals. ${ }^{5}$

In this context, sickle cell anaemia stands out and is a chronic condition with diverse 
clinical manifestations that can lead to recurring hospitalisations and death. Adequate healthcare with a specialised multidisciplinary team and social support can contribute to a reduction in the number of hospitalisations and an improvement in the quality of life of affected individuals. ${ }^{6}$ Patients with sickle cell anaemia experience chronic pain that is treated with opioids, making them a vulnerable population. ${ }^{7}$

Therefore, the use of a valid, reliable assessment tool for measuring the risk of behaviour suggestive of opioid abuse is an important monitoring strategy that can help guide health professionals in the management of these patients. Moreover, evidence produced from such investigations can be used to help healthcare professionals also monitor other types of patients.

Although two systematic reviews have been found that evaluate instruments for measuring risk of opioid abuse, ${ }^{89}$ the current proposal for a systematic review differs in important methodological aspects: (1) more comprehensive search in other databases and use of reverse search strategy, with no time limit and no language restriction; (2) only methodological studies of primary data were included; and (3) proposes a rigorous evaluation of the psychometric properties of the Consensus-Based Standards for the Selection of Health Measurement Instruments (COSMIN), besides including aspects related to the clinical application of these instruments.

In this context, the proposed systematic review has considerable clinical implications with regard to assisting health professionals in the choice of assessment tools that are appropriate to the profile of their patients, since an understanding of the risk of behaviour suggestive of opioid abuse can assist in decision-making and in the adequate management of adult patients with chronic non-cancer pain. Thus, the following research question was posed to guide the analysis of evidence: Do the instruments for measuring the risk of behaviour suggestive of opioid abuse of adults have adequate psychometric properties? The proposed study will involve a systematic review of the literature on the psychometric properties of tools for measuring the risk of behaviour suggestive of opioid abuse in adults with non-cancer pain.

\section{METHODS/DESIGN}

\section{Design and registration of the study}

The present review protocol is registered with the International Prospective Register of Systematic Reviews (PROSPERO). The report on the methods for the review protocol was drafted in accordance with the Preferred Reporting Items for Systematic Review and Meta-Analysis Protocols. ${ }^{10}$ The report on the methods for the systematic review article will follow the Preferred Reporting Items for Systematic Reviews and Meta-Analyses (PRISMA) guidelines. $^{11}$

\section{Patient and public involvement}

It is a systematic review protocol article. We do not work with patients or their companions.

\section{Inclusion of articles}

The following will be the inclusion criteria for the selection of articles: (1) validation studies; (2) assessment tools designed for quantitative evaluation of the risk of behaviour suggestive of opioid abuse; (3) assessment tools designed for evaluation of adults with chronic non-cancer pain; (4) assessment tools designed for adult groups; (5) assessment tools designed for quantitative evaluation of the risk of behaviour suggestive of opioid abuse based on self-reported information from patients; and (6) articles describing the psychometric properties of the tools. No restrictions will be imposed with regard to language or year of publication. Systematic reviews will be excluded.

\section{Search strategy}

The search strategy will be based on the Population Intervention Comparator Outcome Setting ${ }^{12}$ method to form the research question, determine the title and choose the keywords. The PubMed, Web of Science, Cochrane, Scopus, and Cumulative Index to Nursing and Allied Health Literature databases will be searched, and the 'snowball' strategy will also be employed.

The following keywords indexed in the MeSH terms and combinations will be used: 'sickle cell disease', 'opioid', 'validation studies', 'opioid related disorders', 'chronic pain' and 'instrument'. The term 'sickle cell disease' was included to locate instruments developed specifically for adults with this disease due to the high incidence of chronic pain and opioid abuse in this population.

To minimise the risk of bias of the individual studies, two independent reviewers will perform analyses of the titles, abstracts and full texts based on the eligibility criteria. In cases of a divergence of opinion regarding the inclusion of a given study, a third reviewer will be consulted. Descriptive analyses will be performed of the characteristics of the studies, participants, psychometric properties and clinical usefulness of the assessment tools.

\section{Screening, data extraction and comparative content analysis}

All results of the database searching will be filed to record the initial search strategy and subsequent modifications. Duplicate articles will only be counted once. Authors will be contacted for further information, when necessary.

Data extraction will involve the use of a chart specifically designed for the proposed study to organise the following data:

1. Information and general characteristics: authors, year of publication, country and sample characteristics.

2. Description of assessment tool: acronym of the measure, domains, number of items, scores and application format.

The data will be subsequently tabulated in a databank created exclusively for the proposed study. 
The content will be compared through meetings between the two reviewers. Divergence of opinion will be resolved by a third reviewer to extract complete information from all manuscripts. A study selection file will be kept to record the references for all studies excluded and the reasons for exclusion. A flow chart will be created showing the article selection process. All relevant data from the studies will be summarised in tables.

\section{Appraisal of the psychometric properties of the selected instruments}

The COSMIN checklist will be used for the appraisal of the psychometric properties of the selected instruments. This checklist has four domains: reliability, validity, responsiveness and interpretability. ${ }^{13-16}$

\section{Evaluation of clinical usefulness of assessment tools}

For an assessment tool that measures a health phenomenon based on the self-report of adult patients to be adopted by health professionals, it needs to be analysed with regard to its interpretability and viability, which are factors that could influence the decision-making of health professionals in clinical practice. ${ }^{17}$ Therefore, the systematic review article will include an evaluation of these assessment tools based on the criteria proposed by Tyson and Brown ${ }^{18}$ listed below:

- Total time required for the administration, analysis and interpretation of the data obtained using the assessment tool: <10 min (3 points); 10-30 min (2 points); $30-60$ min (1 point); $>1$ hour ( 0 point).

- Cost involved in the acquisition and use of the assessment tool: <£100 (3 points); £100-500 (2 points); $£ 500-1000$ (1 point); £1000 (0 point).

- Need for training and calibration for use of the assessment tool: none ( 2 points); yes, but simple and clinically viable ( 1 point); yes and not clinically viable/ unknown (0 point).

- Portability of the tool (can it be taken to the patient?): yes, easily (fits in pocket) (2 points); yes (fits in a carrying case) (1 point); no or very difficult ( 0 point).

- Accessibility of the tool (are detailed instructions for use available?): yes (complete operating procedure/ instruction manual can be obtained in the article or site) (2 points); no, but the operation can be performed simply based on the description in the article (1 point); no available instructions for use (0 point).

\section{Data synthesis}

The systematic review report will be drafted in accordance with the PRISMA recommendations, ${ }^{11}$ and the certainty of the evidence will be analysed using the Grading of Recommendations Assessment, Development and Evaluation. ${ }^{19}$ For the proposed review, assessment tools with the following qualities will be considered adequate:

- Those with a methodology considered 'good' or 'excellent' based on the COSMIN checklist. ${ }^{13-16}$
- Those with a score of 10 or more points on the Clinical Usefulness Evaluation Scale proposed by Tyson and Brown. ${ }^{18}$

\section{ETHICS AND DISSEMINATION}

The general aim of the review is to provide a discussion on the strong points and limitations of different assessment tools used for measuring the risk of behaviour suggestive of opioid abuse through an analysis of the general characteristics, psychometric properties and clinical usefulness of the measures, as well as the methodological quality of the studies included in the review. This is expected to assist health professionals in determining what measures are more appropriate based on the characteristics of their patients, as well as assist in decision-making processes and determining the most adequate care management for patients with chronic non-cancer pain. The use of valid and reliable instruments is fundamental to the reliability of the evidence produced on a health phenomenon. ${ }^{20}$

In conclusion, the proposed systematic review will provide relevant clinical evidence on the assessment tools designed for measuring the risk of behaviour suggestive of opioid abuse that health professionals can use in the clinical management of patients with chronic non-cancer pain.

The report on the methods for the systematic review article will follow the guidelines of the PRISMA and will be submitted to a peer-reviewed journal. This protocol and the proposed systematic review are activities related to the obtainment of the main researcher's doctoral degree, and her doctoral theses fulfilled all these ethical issues.

\section{Author affiliations}

${ }^{1}$ Neuropsychiatry and Behavioral Sciences, Federal University of Pernambuco, UFPE, Recife, Pernambuco, Brazil

${ }^{2}$ Neuropsychiatry and Behavioral Sciences, Federal University of Pernambuco, UFPE, Recife, Pernambuco, Brazil

${ }^{3}$ Polytechnic School of Pernambuco, University of Pernambuco, UPE, Recife,

Pernambuco, Brazil

${ }^{4}$ Neuropsychiatry and Behavioral Sciences, Federal University of Pernambuco, UFPE, Recife, Pernambuco, Brazil

${ }^{5}$ Neuropsychiatry and Behavioral Sciences, Federal University of Pernambuco, UFPE, Recife, Pernambuco, Brazil

${ }^{6}$ Nursing in Public Health, Federal University of Paraiba, UFPB, João Pessoa, Paraíba, Brazil

Contributors All authors made substantial contributions to the conception and design of the study and participated in the drafting of the submission request. SRG, TdPSdS and SCV conceived of the study, developed the criteria, performed the literature search and selection of the studies, and wrote the present systematic review protocol study. MHdNM, CEdSLR and MDdCL served as advisers throughout all phases of this systematic review protocol study and performed a critical revision of the manuscript. All authors read and approved the final version.

Funding This research received no specific grant from any funding agency in the public, commercial or not-for-profit sectors.

Competing interests None declared.

Patient consent Not required.

Provenance and peer review Not commissioned; externally peer reviewed.

Open access This is an open access article distributed in accordance with the Creative Commons Attribution Non Commercial (CC BY-NC 4.0) license, which 
permits others to distribute, remix, adapt, build upon this work non-commercially, and license their derivative works on different terms, provided the original work is properly cited, appropriate credit is given, any changes made indicated, and the use is non-commercial. See: http://creativecommons.org/licenses/by-nc/4.0/.

\section{REFERENCES}

1. Vowles KE, McEntee ML, Julnes PS, et al. Rates of opioid misuse, abuse, and addiction in chronic pain: a systematic review and data synthesis. Pain 2015;156:569-76.

2. Setnik B, Roland CL, Sommerville KW, et al. A multicenter, primary care-based, open-label study to identify behaviors related to prescription opioid misuse, abuse, and diversion in opioidexperienced patients with chronic moderate-to-severe pain. $J$ Pain Res 2015;8:361.

3. Hser YI, Mooney LJ, Saxon AJ, et al. Chronic pain among patients with opioid use disorder: Results from electronic health records data. J Subst Abuse Treat 2017;77:26-30.

4. Hah J, Sturgeon J, Zocca J, et al. Factors associated with prescription opioid misuse in a cross-sectional cohort of patients with chronic non-cancer pain. J of Pain Research 2017;10:979-87.

5. Stumbo SP, Yarborough BJ, McCarty D, et al. Patient-reported pathways to opioid use disorders and pain-related barriers to treatment engagement. J Subst Abuse Treat 2017;73:47-54.

6. Figueiredo JO, Santos C. Morbidity and mortality due to sickle cell disease in Salvador, Thesis presented to the Post-Graduation Program in Collective Health. Mota, Bahia: Community Health Federal University of Bahia, 2017.

7. Miranda FP, Brito MB. Multidisciplinary care for patients with sickle cell anemia in hospitalized seizures: an integrative review. Revista Enfermagem Contemporânea 2016;5:143-50.

8. Chou R, Fanciullo GJ, Fine PG, et al. Opioids for chronic noncancer pain: prediction and identification of aberrant drug-related behaviors: a review of the evidence for an American Pain Society and American Academy of Pain Medicine clinical practice guideline. J Pain 2009;10:131-46.

9. Lawrence R, Mogford D, Colvin L. Systematic review to determine which validated measurement tools can be used to assess risk of problematic analgesic use in patients with chronic pain. $\mathrm{Br} \mathrm{J}$ Anaesth 2017;119:1092-109.

10. Moher D, Shamseer L, Clarke M, et al. Preferred reporting items for systematic review and meta-analysis protocols (PRISMA-P) 2015 statement. Syst Rev 2015;4:1.

11. Liberati A, Altman DG, Tetzlaff J, et al. The PRISMA statement for reporting systematic reviews and meta-analyses of studies that evaluate healthcare interventions: explanation and elaboration. BMJ 2009;339:1-28.

12. Centre for Reviews and Dissemination. Systematic reviews: CRD's guidance for undertaking reviews in health care. York: University of York, 2009. (accessed 01 Nov 2017).

13. Mokkink LB, Terwee CB, Patrick DL, et al. The COSMIN study reached international consensus on taxonomy, terminology, and definitions of measurement properties for health-related patientreported outcomes. J Clin Epidemiol 2010;63:737-45.

14. Mokkink LB, Terwee CB, Patrick DL, et al. The COSMIN checklist for assessing the methodological quality of studies on measurement properties of health status measurement instruments: an international Delphi study. Qual Life Res 2010;19:539-49.

15. Mokkink LB, Terwee CB, Gibbons E, et al. Inter-rater agreement and reliability of the COSMIN (COnsensus-based Standards for the selection of health status Measurement Instruments) checklist. BMC Med Res Methodol 2010;10:82.

16. Mokkink LB, Terwee CB, Stratford PW, et al. Evaluation of the methodological quality of systematic reviews of health status measurement instruments. Qual Life Res 2009;18:313-33.

17. Martins JC, Aguiar LT, Nadeau S, et al. Measurement properties of self-report physical activity assessment tools in stroke: a protocol for a systematic review. BMJ Open 2017;7:1-5.

18. Tyson SF, Brown P. How to measure fatigue in neurological conditions? A systematic review of psychometric properties and clinical utility of measures used so far. Clin Rehabil 2014;28:804-16.

19. Atkins $D$, Best $D$, Briss PA, et al. Grading quality of evidence and strength of recommendations. BMJ 2004;328:1490.

20. Yang F, Dawes P, Leroi I, et al. Measurement tools of resource use and quality of life in clinical trials for dementia or cognitive impairment interventions: protocol for a scoping review. Syst Rev 2017;6:1-4. 